\title{
Os novos anticoagulantes orais na profilaxia de acidente vascular cerebral em pacientes com fibrilação atrial não valvar
}

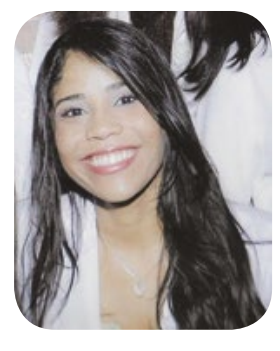

\author{
Itana Naiara Costa Ribeiro ${ }^{1}$, Thiago Santana Santos Queiroz ${ }^{1}$, \\ Matheus Mendes Pires ${ }^{1}$, Pedro Antônio Pereira de Jesus ${ }^{1}$
}

\section{RESUMO}

A warfarina é uma terapia eficaz para profilaxia de eventos cardioembólicos e tromboembólicos em pacientes que sofrem de FA (Fibrilação Atrial) não valvar. Contudo, existem algumas peculiaridades que frequentemente prejudicam a efetividade desse medicamento. Nesse contexto, emergiram os NOACs (Novos Anticoagulantes Orais), dabigatrana, rivaroxabana, apixabana e edoxabana, com a proposta de não inferioridade e possível maior segurança em relação aos AVK (Antagonistas da Vitamina K) para profilaxia de Acidente Vascular Cerebral Isquêmico (AVCl) em pacientes com FA. Nesse artigo de atualização, foram expostos os mecanismos de ação, os estudos que comprovaram a eficácia e segurança dos AODs (Anticoagulantes Orais Diretos), bem como suas limitações. E a partir das evidências disponíveis, foram expostos os perfis dos pacientes que melhor se beneficiarão da terapia com cada um dos NOACs. Por fim, apesar da grande evolução que os AODs representam na profilaxia do AVC em FA, e do grande volume de dados sendo continuamente gerados por diversos ensaios clínicos, ainda há importantes quesitos sobre o uso dessas drogas em determinadas circunstâncias clínicas que precisam de respostas com melhor nível de evidência científica.

PALAVRAS-CHAVE: novos anticoagulantes orais, anticoagulantes orais diretos, fibrilação atrial.

KEY-WORDS: new oral anticoagulants, direct oral anticoagulants, atrial fibrillation.

\section{INTRODUÇÃO}

Desde 1954, a warfarina vem sendo usada em seres humanos e reinou quase absoluta como anticoagulante oral durante cerca de 50 anos, por ser comprovadamente uma terapia eficaz e segura para tratamento e profilaxia de eventos cardioembólicos e tromboembólicos. O uso dos AVKs para prevenção de AVCl em indivíduos com FA mudou completamente as diretrizes e o manejo terapêutico desse grupo de pacientes. A FA é uma arritmia cardíaca comum ${ }^{1,2}$, sendo o AVCI a consequência mais frequente dos fenômenos embólicos causados por ela. A FA chega a ser responsável por cerca de 15 a $20 \%$ das causas de $\mathrm{AVCl}^{3,4,5}$, além de estar associada a um aumento do risco de isquemia cerebral em cerca de 5 vezes $^{1,2}$. Apesar da histórica importância da warfarina, existem algumas limitações inerentes ao seu uso que podem tornar o tratamento, muitas vezes, laborioso. Tais inconvenientes seriam a necessidade de rigorosa monitorização laboratorial da janela terapêutica, controle dietético, interação com vários fármacos, tempo longo para início de ação, bem como tempo incerto para cessação do efeito anticoagulante após suspensão, além de associação com sangramentos graves. Essas limitações chamaram atenção para a necessidade de terapias mais modernas e de manipulação mais prática, abrindo caminho para a era dos NOACs. Os inibidores diretos da coagulação são medicamentos que inibem uma enzima específica na cascata de coagulação. Os agentes atualmente disponíveis agem inibindo diretamente a trombina (fator Ila) ou o fator $\mathrm{Xa}^{5}$. Desde 2010, a US FDA (United States Food and Drug Administration) vem aprovando promissores anticoagulantes orais, quais sejam dabigatrana (Pradaxa $\left.{ }^{\circledR}\right)$, apixabana (Eliquis $\left.{ }^{\circledR}\right)$, rivaroxabana (Xarelto ${ }^{\circledR}$ ) e edoxabana (Savysa ${ }^{\circledR}$ ) para profilaxia do $\mathrm{AVCl}$ entre pacientes com FA não valvar ${ }^{2}$ (Tabela 1). Nesse contexto, essas novas drogas vêm comprovando a não inferioridade em relação à veIha warfarina, inclusive, com maior segurança, além de eficácia pelo menos equivalente na prevenção de eventos cardioembólicos. Contudo, apesar de suas muitas vantagens (rápido início de ação, menos intera- 
ções medicamentosas e não necessidade de controle dietético ou de faixa terapêutica), essas terapias não são perfeitas, e vêm associadas a limitações quanto ao uso em situações pontuais, seja por dados insuficientes que embasem o seu uso, seja em certas comorbidades, como disfunção renal e Síndrome da Imunodeficiência Adquirida (SIDA) ${ }^{5}$. E para a infelicidade universal, ainda não há antídotos específicos em caso de eventos hemorrágicos graves para os inibidores do fator Xa. Pela ordem cronológica que foram surgindo, os AODs serão apresentados e discutidos, inicialmente em tópicos individuais e na sequência em pontos comuns comparativos com a warfarina.

\section{ANTICOAGULANTES NÃO ANTAGONISTAS DA VITAMINA K}

\section{Dabigatrana}

A molécula da dabigatrana é conhecida desde 1980, mas foi somente em 2010 que a FDA aprovou seu uso para profilaxia de AVC isquêmico no contexto de FA. Esse anticoagulante atua como inibidor direto potente, competitivo e reversível da trombina ou fator Ila (uma serina protease responsável pela conversão de fibrinogênio em fibrina na cascata de coagulação). A dabigatrana inibe a trombina livre e fibrinada, evitando assim a formação de trombo ${ }^{6}$. Com biodisponibilidade de $6,5 \%$, sua meia-vida no soro é de aproximadamente 12 a 17 horas $^{7}$, o efeito anticoagulante máximo ocorre dentro de 2 a 3 horas após a ingestão e $80 \%$ da dose administrada é excretada pelos rins ${ }^{4}$. A depuração da dabigatrana é de $100 \mathrm{ml} / \mathrm{min}$. A exposição à dabigatrana aumenta em indivíduos com disfunção renal. A farmacocinética e a farmacodinâmica não são afetadas por disfunção hepática moderada (classe $B$ de Child-Pugh) após uma única dose oral de 150mg. A exposição é aumentada em idosos, mas a farmacocinética parece não diferir entre etnias ${ }^{6}$. Seu uso não precisa de monitorização laboratorial de rotina, mas, para situações específicas, é importante saber que a droga prolonga o tempo de tromboplastina parcial ativada, tempo de trombina e o tempo de coagulação de ecarina. No efeito máximo nos parâmetros de coagulação ocorre simultaneamente a concentração plasmática máxima, dentro de 2 horas de administração, e os efeitos farmacodinâmicos diminuem em paralelo com o declínio das concentrações plasmáticas. A redução da concentração plasmática pode ocorrer dentro de 4 a 6 h, após atingir o pico máximo ${ }^{6,8}$.

Em setembro de 2009, foi publicado o estudo RE-LY (Dabigatran versus Warfarin in Patients with Atrial
Fibrillation), que tinha por objetivo avaliar a não inferioridade da dabigatrana em relação à warfarina. Os resultados do RE-LY e de outros estudos vêm mostrando menor risco de mortalidade geral, AVC isquêmico e AVC hemorrágico em relação à warfarina ${ }^{9}$. Inclusive maior dosagem foi associada a um risco significativamente menor de ACV ou embolia sistêmica, sem diferença significativa para o risco de sangramentos graves ${ }^{6}$. Contudo, alguns dados têm identificado maiores taxas de sangramento gastrointestinal no grupo dabigatrana, na dose de 150mg, 2 vezes por dia. Uma metanálise mostrou que o risco de sangramento gastrointestinal em pacientes recebendo $150 \mathrm{mg}$ de dabigatrana 2 vezes por dia, em comparação com warfarina, seria maior entre aqueles com idade superior a $65 \operatorname{anos}^{10}$. Outra revisão sistemática comparando dabigatrana com warfarina, que incluiu mais de 27.000 pacientes, encontrou que a mortalidade em 30 dias foi menor com a dabigatrana ${ }^{11}$. Um ensaio clínico com asiáticos apresentou resultados semelhantes aos até então vistos em outros estudos em relação à eficácia, além de não ter confirmado maior risco de sangramento gastrointestinal grave ou infarto agudo do miocárdio (IAM), comparado à warfarina ${ }^{12}$. Outros dados observacionais sugerem menor risco de recorrência de sangramento com a dabigatrana em relação à warfarina após retomada da anticoagulação suspensa por evento hemorrágico, com melhor risco-benefício também para dabigatrana ${ }^{12}$. Além disso, parece que a dabigatrana estaria associada a menores taxas de fratura osteoporótica ${ }^{13}$ e de sangramento grave em pacientes diabéticos ${ }^{8}$. Apesar desses resultados promissores, ainda restava a limitação da ausência de antídoto em caso de sangramentos graves. Em agosto de 2015, foi publicado o RE-VERSE AD (Idarucizumab for Dabigatran Reversal), que apresentou ao mundo o efeito do antídoto idaracizumab. O idarucizumab é um fragmento de anticorpo monoclonal que se liga à dabigatrana ligada à trombina, neutralizando assim sua atividade, é indicado em casos de sangramentos graves com risco iminente de morte. ${ }^{14}$

A dose indicada de dabigatrana para profilaxia de AVC em FA é $300 \mathrm{mg} / \mathrm{dia}$ (150mg, 2x/dia), em situações específicas (idade $>80$ anos, uso de verapamil $150 \mathrm{mg} /$ dia, $\mathrm{CICr}$ [clearance de creatinina] entre 30 e $50 \mathrm{ml} / \mathrm{min}$, uso concomitante de dronedarone ou cetaconazol) deve-se reduzir a dose para $220 \mathrm{mg} / \mathrm{dia}(110 \mathrm{mg} 2 x / \mathrm{dia})^{4}$. A dabigatrana deve ser usada com cautela na disfunção renal, sendo contraindicada naqueles que fazem uso de inibidores da P-gp (P-glicoproteína) com $\mathrm{ClCr}$ $<30 \mathrm{ml} / \mathrm{min}$ e em $\mathrm{ClCr}<15 \mathrm{ml} / \mathrm{min}^{4}$. Interações medica- 
mentosas com a dabigatrana surgem principalmente pelo envolvimento da P-gp. Por exemplo, a rifampicina é um indutor do CYP3A e estudos mostram que também pode induzir a expressão da $\mathrm{P}-\mathrm{gp}^{15}$. A rifampicina causa redução da exposição da dabigatrana, dessa forma, a orientação é que o uso simultâneo deve ser evitado ${ }^{16}$. Por outro lado, inibidores da P-gp, tais como cetoconazol e dronderone, aumentam a exposição da dabigatrana em caso de disfunção renal moderada (ClCr entre 30 a $50 \mathrm{ml} / \mathrm{min}$ ), por isso, nessa situação, deve-se reduzir a dose de dabigatrana para $75 \mathrm{mg} 2 \mathrm{x} /$ dia $^{16}$. Já em caso de disfunção renal grave, $(\mathrm{ClCr}$ de $15 \mathrm{a} 30 \mathrm{ml} / \mathrm{min}$ ), o uso ao mesmo tempo de dabigatrana com cetoconazol ou dronderone, ou de outros inibidores da P-gp (verampamil, amiodarona, quinidina, claritromicina e ticagrelor), deve ser evitado ${ }^{16}$.

\section{Rivaroxabana}

A rivaroxabana é um inibidor direto do fator $\mathrm{Xa}$, com biodisponibilidade de $70 \%$, sua meia-vida sérica varia de 5 a 9 horas em voluntários saudáveis, já em idosos a meia-vida varia de 11 a 13 horas, dois terços da dose é metabolizada no fígado ( $1 / 2$ da excreção por via renal e $1 / 2$ via hepatobiliar nas fezes $)^{4,17}$. Em setembro de 2011, foi publicado o ROCKET-AF (Rivaroxaban Once Daily Oral Direct Fator Xa Inhibition Compared with Vitamin K Antagonism for Prevention of Stroke and Embolism Trial in Atrial Fibrilation) e os resultados sugeriram a não inferioridade da rivaroxabana em relação à warfarina para a prevenção de AVC ou embolia sistêmica no contexto de FA. Neste trabalho, não houve diferença significativa entre os grupos estudados em relação a risco de hemorragia grave, embora hemorragia intracraniana e hemorragia fatal ocorreram menos frequentemente no grupo rivaroxabana ${ }^{17}$. Uma metanálise mostrou taxas de sangramento semelhantes com uso de rivaroxabana em relação à warfarina $^{10}$. A rivaroxabana, bem como os demais inibidores diretos do fator $\mathrm{Xa}$, ainda não dispõe de antagonista específico para reversão do efeito anticoagulante em situações de sangramento grave com risco de morte, as medidas que devem ser tomadas atualmente para esse tipo de evento serão discutidas posteriormente, em tópico específico. Existem restrições do uso em disfunção renal, a dose usual indicada é $20 \mathrm{mg} /$ dia para pacientes com $\mathrm{ClCr}>50 \mathrm{ml} / \mathrm{min}$, ou $15 \mathrm{mg} / \mathrm{dia}$ se ingerido com refeições noturnas em paciente com $\mathrm{CICr}$ entre 15 e $50 \mathrm{ml} / \mathrm{min}$, se $\mathrm{ClCr}<15 \mathrm{ml} / \mathrm{min}$, este medicamento não deve ser usado 4 .

Interações podem ocorrer entre a rivaroxabana e a combinação de indutores de P-gp com fortes indutores de CYP3A4 (rifampicina, carbamazepina, fenitoína), estes agem reduzindo a exposição e a eficácia da rivaroxabana, dessa forma, o uso concomitante deve ser evitado ${ }^{16}$. A interação também pode ocorrer na combinação de inibidores de P-gp com fortes inibidores de CYP3A4 (cetoconazol, itraconazol, inibidores da protease do HIV [ritonavir, lopinavir / ritonavir, indinavir], conivapta) em uso concomitante com rivaroxabana, pois eles reduzem a exposição da rivaroxabana, assim, o uso simultâneo com essas drogas não é aconselhado ${ }^{16}$. Em relação à combinação de inibidores de P-gp com inibidores moderados de CYP3A4 (diltiazem, verapamil, amiodarona, dronedarona, eritromicina), o efeito da associação com a rivaroxabana causaria aumento da exposição dela nos pacientes com disfunção renal, contudo, se $\mathrm{ClCr}$ entre 15 e $80 \mathrm{ml} / \mathrm{min}$, a rivaroxabana não deve ser usada concomitantemente, a menos que os riscos-benefícios justifiquem. Nenhuma evidência de interação foi observada no ROCKET AF entre a rivaroxabana e combinações de inibidores da P-gp com inibidores moderados de 3A4 (amiodarona, diltiazem e verapamil) ${ }^{16}$.

\section{Apixabana}

A apixabana é um inibidor direto do factor $\mathrm{Xa}$, de rápida absorção, a excreção ocorre principalmente através do fígado, mas $25 \%$ da excreção é renal ${ }^{4,18}$. A biodisponibilidade é de $50 \%$, a meia-vida no soro varia de 8 a 15 horas $^{18}$. A dose recomendada é de $5 \mathrm{mg}, 2$ vezes ao dia e deve ser reduzida para $2,5 \mathrm{mg}, 2$ vezes ao dia, se há dois desses três critérios: idade $\geq 80$ anos, $\leq 60 \mathrm{~kg}$, se o nível de creatinina sérica $\geq 1,5 \mathrm{mg} /$ $\mathrm{dl}$ ou insuficiência renal grave $(\mathrm{CrCl} 15 \text { a } 29 \mathrm{ml} / \mathrm{min})^{18}$. Em setembro de 2011, foi publicado o ARISTOTLE (Apixaban versus Warfarin in Patients with Atrial Fibrillation), cujo objetivo era determinar se a apixabana era não inferior à warfarina para redução da taxa de AVC ou embolia sistêmica entre pacientes com FA. O ARISTOTLE conseguiu mostrar superioridade da apixabana, comparada à warfarina na prevenção de AVC ou embolia sistêmica, causando menor sangramento e resultando em menor mortalidade ${ }^{18}$. Uma metanálise também mostrou menor sangramento com o uso de apixabana em relação à warfarina ${ }^{10}$. A dose indicada é $10 \mathrm{mg} /$ dia (5mg $2 x /$ dia), a redução da dose para $5 \mathrm{mg} /$ dia $(2,5 \mathrm{mg} 2 \times /$ dia) seria indicada em situações específicas (idade $>80$ anos, peso $<60 \mathrm{Kg}$, creatinina sérica $>1,5 \mathrm{mg} / \mathrm{dl}$ ou $\mathrm{ClCr}$ entre $15 \mathrm{e} 29 \mathrm{ml} /$ minuto $)^{4}$.

Interações ocorrem com indutores fortes de P-gp e de CYP3A4 (rifampicina, carbamazepina, fenitoína), podendo reduzir a exposição da apixabana, então o 
uso concomitante deve ser evitado ${ }^{16}$. Interações com inibidores fortes de P-gp e de CYP3A4 (cetoconazol, itraconazol, ritonavir e claritromicina) aumentam a exposição da apixabana. Nesse caso, se a dose em uso é de $5 \mathrm{mg}$ ou $10 \mathrm{mg} 2 \mathrm{x} / \mathrm{dia}$, deve-se reduzi-la em $50 \%$, quando há coadministração. Já na dose de 2,5mg, 2 vezes ao dia, deve-se evitar coadministração ${ }^{16}$.

\section{Edoxabana}

A edoxabana é um inibidor do factor $\mathrm{Xa}$, de uso diário, excretado com $62 \%$ de biodisponibilidade e tem uma meia-vida de 10 a 14 horas, atinge máxima concentração em 1 a 2 horas. Sua eliminação ocorre $50 \%$ por via renal e $50 \%$ por via hepática. Existe restrição de dose em caso de $\mathrm{ClCr}$ entre 15 e $50 \mathrm{ml} /$ minuto. Nessa situação, deve ser reduzida para $30 \mathrm{mg} / \mathrm{dia}$. A dose indicada para prevenção do AVC é de $60 \mathrm{mg} / \mathrm{dia}^{4}$. $O$ estudo EDOXABAN AF (Effective Anticoagulation With Factor Xa Next Generation ion Atrial Fibrilation Trial), publicado em novembro de 2013, buscou avaliar a não inferioridade da droga em relação à warfarina, este ensaio clínico conseguiu cumprir seu objetivo ${ }^{19}$. Uma metanálise sugeriu menor risco de sangramento com o uso de edoxabana em relação à warfarina ${ }^{10}$. Outro estudo mostrou que doses diárias de edoxabana (60mg ou $30 \mathrm{mg}$ ) foram mais seguras do que quando administradas 2x/dia. Apesar desses dados, a eficácia e segurança, a longo prazo, da edoxabana, em comparação com a warfarina, em pacientes com $\mathrm{FA}$, ainda não é conhecida. A simulação farmacocinética mostrou que pacientes com baixo peso corporal, disfunção renal moderada ou grave, ou uso concomitante de um potente inibidor da $\mathrm{P}$-gp, são indicações para redução da dose da edoxabana em $50 \%{ }^{19}$. A dose de edoxabana indicada é $60 \mathrm{mg} / \mathrm{dia}$, se $\mathrm{ClCr}$ entre 50 e $95 \mathrm{ml} /$ minuto; 30mg/dia, se $\mathrm{ClCr}$ entre 15 e $50 \mathrm{ml} / \mathrm{minuto}^{4}$; e não deve ser usado com $\mathrm{CICr}>95 \mathrm{ml} /$ minuto (conforme estimado pela equação de Cockcroft-Gault, foi identificada uma maior taxa de AVC isquêmico neste grupo, quando em uso de edoxabana) $)^{5}$.

As interações podem ocorrer com indutores da P-gp (rifampicina), estes reduzem a exposição da edoxabana. Assim, o uso concomitante deve ser evitado ${ }^{16}$. Os fortes inibidores de P-gp (ritonavir, nelfinavir, saquinavir, indinavir e ciclosporina) aumentam a exposição da edoxabana, nesse caso, deve-se evitar o uso concomitante em pacientes que tomam edoxabana para tratamento de TEV (tromboembolismo venoso) ${ }^{16}$. Os inibidores de P-gp (verapamil, quinidina, azitromicina, claritromicina, itraconazol e cetoconazol) aumentam a exposição da edoxabana, deve-se reduzi-la para $30 \mathrm{mg} / \mathrm{dia}$ no uso concomi- tante com essas drogas para tratamento de TEV, mas não é recomendada a redução da dose se a indicação do uso de edoxabana for $F^{16}$. No ENGAGE AF, a redução na dose de edoxabana pelo uso concomitante com inibidores de P-gp (verapamil, quinidina e dronedarona) foi associada com aumento no risco relativo de AVC ou embolia sistêmica em relação à warfarina ${ }^{20}$.

\section{DADOS COMPARATIVOS ENTRE OS NOACS E A WARFARINA}

\section{Redução de AVC e hemorragia grave}

Desde que os NAOCs foram aprovados pela FDA, algumas metanálises vêm sendo realizadas com os dados dos quatro principais estudos que embasaram o uso dessas drogas $7,17,18,19$. Uma dessas metanálises, abrangendo mais de 72.000 desses pacientes, mostrou que os NAOCs são pelo menos tão efetivos quanto a warfarina para prevenção de AVC em FA não valvar, com a vantagem de menor ocorrência de sangramentos graves. O risco de sangramento intracraniano (incluindo AVC hemorrágico) é reduzido em mais que a metade em relação aos AVKs. O que chama atenção é o risco de sangramento gastrintestinal, que, segundo as análises, pode ocorrer até $25 \%$ a mais com o uso de NAOCs. A redução do risco relativo de morte é por volta de $10 \%$. As descobertas dos quatro principais ensaios foram apoiadas por análises observacionais de registro ${ }^{3}$. Outra metanálise encontrou que os AODs tiveram um perfil de risco favorável, com reduções significativas em AVC, hemorragia intracraniana e mortalidade, e com risco de hemorragia grave semelhante a da warfarina, porém com aumento do sangramento gastrointestinal ${ }^{21}$. Uma terceira metanálise, com mais de 100.000 pacientes, mostrou que a taxa de letalidade para hemorragia grave foi de $7,6 \%$ com NAOCs, $11 \%$ com warfarina, a diferença foi estatisticamente significante. Também ocorreu redução no risco relativo de sangramento fatal, de mortalidade cardiovascular e de mortalidade por todas as causas ${ }^{5}$. Não há testes randomizados comparando os quatro NAOCs entre si em relação à segurança e à eficácia, embora estudos observacionais tenham mostrado diferenças no risco de eventos hemorrágicos entre diferentes NOACs.

\section{Principais causas de morte entre pacientes com FA em uso de anticoagulantes orais}

As causas e riscos de mortalidade são alguns dos eventos mais monitorados no seguimento dos pacientes com FA em anticoagulação para profilaxia de AVC. Uma metanálise com os principais trials sobre NAOCs 
encontrou que a principal causa da morte em pacientes com FA anticoagulados seria a doença cardíaca, correspondendo a cerca de $46 \%$ da mortalidade (morte súbita, insuficiência cardíaca e IAM), enquanto que espantosamente o AVC isquêmico e sangramentos fatais representariam apenas aproximadamente $6 \%$ de todas as causas de morte nesse grupo. $O$ uso de estatina, bem como os tratamentos adequados de insuficiência cardíaca e doença arterial coronariana (DAC), foi associado à menor mortalidade ${ }^{1}$. Esses dados reforçam a importância do controle de comorbidades e fatores de risco para reduzir as taxas de morte nesse perfil de pacientes. A partir desses dados, pode-se inferir que apenas a anticoagulação isoladamente é provavelmente menos efetiva para reduzir mortalidade do que quando associada ao controle adequado de insuficiência cardíaca, DAC, diabetes e mudanças no estilo de vida. Em relação às causas de morte potencialmente evitáveis, o sangramento pós-traumático foi a principal causa. Além disso, o risco de hemorragia grave associada ao trauma em pacientes anticoagulados com NAOCs foi menor em relação ao grupo que fazia uso de warfarina. De todos os sangramentos fatais, o principal componente contabilizado foi o AVC hemorrágico. Quando comparado NAOCs com a warfarina, foi encontrado risco reduzido de sangramento fatal (incluindo menor risco de sangramento intracraniano), de mortalidade cardiovascular e de mortalidade por todas as causas no grupo dos pacientes em uso dos AODs ${ }^{1}$.

\section{Custo-efetividade}

É sabido que os NAOCs têm o valor de mercado mais elevado que AVKs. E por isso, apesar das muitas vantagens dos AODs, a warfarina ainda persiste, sendo preferida pelo baixo custo, principalmente em programas governamentais de distribuição de medicamentos. Nesse contexto, estudos têm sido realizados com o objetivo de avaliar os reais gastos com a anticoagulação, associando o preço de compra das drogas com situações do "mundo real", e análises comparativas de custo-efetividade dos NAOCs entre si e individualmente com a warfarina têm sido realizadas. Uma dessas pesquisas mostrou que a apixabana foi uma estratégia mais rentável do que dabigatrana, edoxanana e rivaroxabana. Contudo, a relação custo-eficácia da apixabana foi sensível à estimativa de eficácia da edoxabana e da dabigatrana ${ }^{2}$, e entre pacientes com alto risco de AVC, com CHADS $2 \geq 3$, a dabigatrana foi o NAOC mais rentável. Da mesma forma, outro estudo identificou que NOACs podem ser opções econômicas em países cujo controle de anticoagulação dos pacientes é difícil de al- cançar com warfarina, sugerindo que a apixabana seria o medicamento substituto com melhor custo-benefício, seguida por dabigatrana, edoxabana e rivaroxabana, respectivamente. Deve-se ter em mente que a relação custo-eficácia dos NOACs é altamente sensível ao controle da anticoagulação com warfarina, pois o descontrole da faixa terapêutica pode levar a gastos imprevistos, desde a realização de exames até internações por desfechos negativos. Em suma, pode-se sugerir que os NOACs, em determinadas circunstâncias, talvez sejam opções mais econômicas ${ }^{22}$.

\section{ESCOLHENDO OS NOACS DE ACORDO COM AS COMORBIDADES}

Diante da emergência de opções múltiplas para profilaxia de AVC em FA, rotineiramente podem aparecer situações de indecisão sobre qual anticoagulante oral seria o mais adequado para o perfil de determinado paciente. Pensando nesse contexto, a Sociedade Europeia de Cardiologia realizou uma revisão com o objetivo de orientar e padronizar as indicações de NOACs e AVKs, priorizando a escolha da droga conforme as principais comorbidades. Segue abaixo um resumo adaptado de alguns tópicos dessas orientações, resultado de análises de subgrupos, a partir dos principais estudos que envolveram os NAOCs ${ }^{23}$.

\section{Doença arterial coronariana estável}

A DAC é uma entidade relativamente comum e esteve presente em até $30 \%$ dos pacientes nos quatro principais estudos com FA e NAOCs. As análises retrospectivas não foram suficientes para esclarecer a existência de uma preferência clara para nenhum dos NAOCs, em relação à warfarina em DAC. Um documento do consenso europeu manifestou preocupação sobre a possibilidade da dabigatrana aumentar o risco de IAM. Contudo, uma grande análise da FDA não encontrou evidências que comprovassem aumento desse risco, em comparação com a warfarina. Conclusão: não há preferência de um NOAC em relação a outro para pacientes com DAC estável e $F A^{15}$.

\section{Doença arterial oclusiva periférica (DAOP)}

Em FA associada à DAOP, os dados disponíveis não permitem preferência de algum NOAC em relação à warfarina. Conclusão: se for escolhido um NOAC, a preferência deve ser a mesma que seria em caso de paciente com DAC estável e $\mathrm{FA}^{23}$.

Intervenção coronariana com colocação de stent $\mathrm{O}$ tratamento medicamentoso do paciente que tem 
FA e DAC, que precisa colocar stent, é complicado pela necessidade de tripla terapia (anticoagulante para prevenção de AVC e dupla antiagregação para prevenção de trombose de stent e eventos coronarianos), pois isso aumenta o risco de sangramentos, incluindo intracranianos. Atualmente, existem alguns estudos comparando o uso da warfarina com os NOACs em pacientes que têm DAC, porém ainda não há evidências robustas sobre superioridade/equivalência de uma terapia em relação a outra. Se um NOAC for escolhido, deve-se preconizar as doses mais baixas testadas ${ }^{23}$. Existem estudos para avaliar o uso de dabigatrana (REDUAL-PCI ${ }^{24}$, rivaroxabana (PIONEER-AF-PCI) ${ }^{25,26}$. E há outro em andamento para avaliar a apixabana (AUGUSTUS NCT02415400) nessa situação. As doses dos NOACs para prevenção de AVC em FA são as seguintes: dabigatrana - 110mg, 2 vezes ao dia; rivaroxabana - 15mg, 1 vez ao dia; apixabana - 2,5mg, 2 vezes ao dia; ou edoxabana - 30mg, 1 vez ao dia. Conclusão: pacientes com FA submetidos à colocação de stent por DAC, e que precisam de tripla terapia, podem usar warfarina ou NOAC, entretanto não há evidência para embasar a preferência de um NOAC sobre o outro.

\section{Cardioversão e ablação por FA}

$\mathrm{O}$ risco de AVC em pacientes adequadamente anticoagulados, submetidos à cardioversão por FA, cai de $5 \%$ a $7 \%$ (sem anticoagulação) para cerca de $1 \%$, quando anticoagulado com warfarina ${ }^{23}$. Análises retrospectivas de ensaios com NOACs não encontraram diferenças em segurança ou eficácia entre as terapias com NOACs ou AVKs em pacientes com FA submetidos à cardioversão ${ }^{7,17,18}$. Existem ensaios clínicos em andamento (EMANATE-NCT02100228 e ENSURE-NCT02072434) que fornecerão informações adicionais sobre a segurança da cardioversão em pacientes que tomam NOACs. Os dados disponíveis sugerem que os NOACs são alternativas seguras e efetivas nesse contexto. Outra questão importante em FA é a atual tendência de realizar anticoagulação durante o procedimento de ablação, parece que existe menor risco tromboembólico quando se mantém o paciente anticoagulado com warfarina ${ }^{27,28}$. Nesse contexto, em relação aos NOACs, estudos com a dabigatrana sugerem que, em relação à segurança e eficácia, ela é semelhante aos AVKs. Outro estudo mostrou menor risco de sangramento com a dabigatrana ${ }^{29,30}$, algumas pesquisas também sugerem que a rivaroxabana e a apixabana podem ser alternativas seguras e eficazes $^{31,24}$. Conclusões: os AVKs são primeira escolha em pacientes com FA submetidos à cardioversão. Se escolhidos os NOACs, não há dados que sustentem a preferência entre apixabana, dabigatrana e rivaroxabana $^{23}$. Em relação à ablação por $\mathrm{FA}$, a primeira escoIha é a warfarina, seguida de dabigatrana, apixabana ou rivaroxabana, e não há dados disponíveis sobre a segurança e eficácia da edoxabana ${ }^{23}$.

\section{Doença de válvula cardíaca e válvula cardíaca protética mecânica}

Os ensaios com NOACs excluíram os pacientes com estenose mitral, pois esta alteração está altamente associada a AVC. Logo, há poucas informações sobre a eficácia de NOACs nesta população. Já outros tipos de doença de válvula cardíaca (insuficiência mitral ou tricúspide e estenose ou insuficiência aórtica) ocorrem frequentemente em pacientes com $F A^{7,17,18,19}$. Conclusão: a warfarina é a primeira escolha em pacientes com FA e válvulas cardíacas protéticas mecânicas ou estenose mitral grave a moderada (reumática), os NOACs não devem ser usados nesses doentes. Por outro lado, no caso de FA associada a outros tipos de anormalidades valvares, apixabana e rivaroxabana podem ser primeira escolha. A segunda escolha seria dabigatrana ou edoxabana, ficando antagonistas da vitamina $\mathrm{K}$ como terceira linha ${ }^{23}$

\section{CHA2DS2VASC com pontuação 1 para homens} e 2 para mulheres

Pacientes com FA e um ou mais fatores de risco adicional para AVC têm um risco aumento para este evento, neste caso a anticoagulação deve ser considerada. Em pessoas com baixa pontuação no CHA2DS2-VASC (1 para homens e 2 para mulheres), os dados de ensaios clínicos são limitados, pois não há ensaios randomizados para estes grupos. Existem alguns dados de análises de subgrupos sobre o uso de dabigatrana e apixabana em pacientes com um fator de risco para AVC que mostraram segurança e eficácia semelhante entre as duas drogas, enquanto que os estudos que avaliaram rivaroxabana e edoxabana recrutaram pacientes com dois ou mais fatores de risco. Conclusão: pacientes com FA e um único fator de risco adicional para AVC, diferente de sexo, dados limitados sugerem que dabigatrana (150mg $2 x /$ dia) é preferida ou apixabana pode ser considerada.

\section{Sangramento gastrointestinal}

Os NOACs têm sido associados com aumento do risco de sangramentos gastrointestinais em relação à warfarina. No estudo RE-LY, a dabigatrana (na dose de $150 \mathrm{mg}$, 2vezes ao dia) teve uma taxa mais elevada de 
sangramento no trato gastrointestinal ${ }^{7}$. O risco foi aumentado em mulheres com $\geq 75$ anos e homens com $\geq$ 85 anos. Por outro lado, com a dose de $75 \mathrm{mg}$ de dabigatrana $2 x /$ dia, o risco de sangramento gastrointestinal foi semelhante ao grupo warfarina. No estudo ROCKET $\mathrm{AF}$, pacientes que receberam rivaroxabana $20 \mathrm{mg} 1 \mathrm{x} / \mathrm{dia}$ tiveram maior risco de sangramento gastrointestinal em relação ao grupo da warfarina, já a incidência de risco de vida e os sangramentos gastrointestinais fatais foram semelhantes nos dois braços do estudo ${ }^{17}$. Ocorreu maior risco nos pacientes com idade $\geq 75$ anos. No ARISTOTLE, os resultados mostraram taxa semelhante de sangramento gastrointestinal no grupo apixabana $5 \mathrm{mg} 2 \mathrm{x} /$ dia e no grupo warfarina ${ }^{18}$. O ENGAGE AF mostrou um risco aumentado com edoxabana na dose de $60 \mathrm{mg} / \mathrm{dia}$ versus warfarina. Por outro lado, na dose de $30 \mathrm{mg} / \mathrm{dia}$, a edoxabana foi associada a menor risco ${ }^{19}$. Conclusão: pacientes com alto risco de sangramento gastrointestinal a primeira escolha seria apixabana, $5 \mathrm{mg}, 2$ vezes ao dia; ou dabigatrana $110 \mathrm{mg}, 2$ vezes ao dia. A segunda escolha seria a dabigatrana, 150mg, 2 vezes ao dia; edoxabana, 60mg/dia; ou rivaroxabana, 20mg, $1 \mathrm{vez}$ ao dia. O risco hemorrágico gastrointestinal da dabigatrana ou da edoxabana é dependente da dose. O aumento do risco de hemorragia gastrointestinal de dabigatrana e rivaroxabana é mais evidente em pacientes a partir de 75 anos de idade ${ }^{32}$.

\section{Insuficiência renal}

Todos os NOACs dependem da depuração renal em algum grau, sendo as respectivas taxas de excreção via renal de aproximadamente 80 a $85 \%$ para dabigatrana, 35 a $50 \%$ para edoxabana, 33 a $66 \%$ para rivaroxabana e 25 a $27 \%$ para apixabana ${ }^{5,16,32}$. Nos doentes com disfunção renal, a maior concentração plasmática dos NOACs aumenta o risco de hemorragia ${ }^{5}$. Em vigência de FA e insuficiência renal estágio III, a apixabana $(5 \mathrm{mg}, 2$ vezes ao dia) pode ser usada. Nas seguintes situações, a dose da apixabana deve ser reduzida para 2,5mg, 2 vezes ao dia: idade $\geq 80$ anos, peso $\leq 60 \mathrm{~kg}$ e creatinina sérica $\geq 1,5 \mathrm{mg} / \mathrm{dl}$. Outras opção são a rivaroxabana (15mg/dia) ou a edoxabana (30mg/dia); como segunda escolha, pode-se usar a dabigatrana (110mg, 2 vezes ao dia). Não são recomendadas dabigatrana nas doses de $150 \mathrm{mg}$, 2 vezes ao dia, rivaroxabana - $20 \mathrm{mg} / \mathrm{dia}$, ou edoxabana - $60 \mathrm{mg} / \mathrm{dia}$, em insuficiência renal, estágio III. Já nos casos de pacientes com FA em hemodiálise, a primeira escolha é, se possível, a não anticoagulação ou terapia com AVK, e não é recomendado NOACS. A primeira escolha para pacientes com $\mathrm{FA} \mathrm{e} \mathrm{CICr}$ de 95 $\mathrm{ml} / \mathrm{min}$ seria a dabigatrana $-150 \mathrm{mg}, 2$ vezes ao dia; rivaroxabana - 20mg/dia ou apixabana - $5 \mathrm{mg}, 2$ vezes ao dia, mas não há evidência para apoiar preferência de algum NOACS sobre a warfarina. Em algumas referências, a edoxabana, $60 \mathrm{mg} / \mathrm{dia}$, é contraindicada em pacientes com $\mathrm{ClCr}>95 \mathrm{ml} / \mathrm{min}^{16}$. Conclusão: deve-se reduzir as doses dos NOACs em paciente com disfunção renal, conforme a faixa de depuração de creatinina ${ }^{4,32}$.

\section{CONDUTAS EM SANGRAMENTOS GRAVES COM NOACS}

Em caso de sangramento grave ou com risco de vida, no qual o indivíduo permaneça ativamente anticoagulado, o agente específico para reverter a anticoagulação está disponível apenas para a dabigatrana, mas não para os inibidores diretos do fator Xa. Existem algumas estratégias para tentar reduzir sangramento baseadas em dados de séries de casos e experiências clínicas. Sugere-se em situações de grave sangramento a remoção de droga da circulação, do trato gastrointestinal e terapias pró-hemostáticas ${ }^{33}$. As terapias mais potentes estão indicadas para sangramentos com risco de morte iminente, como o idarucizumab e os concentrados de complexos de protrombina ativados ou não ativados, pelo risco potencial de causar eventos trombóticos ${ }^{5}$.

\section{Sangramento por uso de inibidor da trombina}

Diante disso, as orientações atuais para reduzir sangramento associado à dabigatrana são as seguintes: 1. Hemorragia grave (sangramento gastrointestinal maciço, retroperitoneal, intracraniano ou compartimental): pode-se usar um agente antifibrinolítico (ácido tranexâmico ou ácido epsilon-aminocaproico), carvão ativado via oral (se a última dose da dabigatrana tiver sido dentro de duas horas) e hemodiálise (casos selecionados). 2. Risco de morte iminente: o idarucizumab na dose de $5 \mathrm{~g}$ pode ser associado; quando indisponível, pode-se usar concentrado de complexo de protrombina ativado. Não vêm sendo usados de forma concomitante o idarucizumab e concentrado de complexo protrombínico, exceto em risco extremo, devido à falta de dados sobre a segurança em relação a possíveis eventos trombóticos. Transfusão de sangue e/ou plaquetas pode ser realizada conforme a necessidade ${ }^{5}$.

\section{Sangramento por uso de inibidor do fator Xa}

No caso de rivaroxabana, apixabana, edoxabana: 1. Hemorragia grave: pode-se usar um agente antifibrinolítico, além de carvão ativado oral, se a última dose do anticoagulante tiver sido recente (rivaroxabana até 
oito horas, apixabana até seis horas e edoxabana até duas horas). No caso dos inibidores diretos do factor Xa a diálise não deve ser realizada. 2. Risco iminente de morte: é orientado uso de complexo protrombínico não ativado com quatro fatores, na dose de 50 unida$\operatorname{des} / \mathrm{Kg}^{5}$.

\section{Andexanet alfa}

Andexanet alfa é uma proteína recombinante inativa, que se liga aos inibidores do fator Xa, foi projetada para reverter os seus efeitos anticoagulantes. Alguns estudos vêm tentando avaliar segurança e eficácia da droga, o ANNEXA-4 (Andexanet Alfa for Acute Major Bleeding Associated with Factor Xa Inhibitors) avaliou 67 voluntários, incluindo pacientes que haviam sofrido sangramento agudo grave dentro de 18 horas após uso de apixabana ou rivaroxabana, concluindo que ocorreu hemostasia efetiva em $79 \%$ da amostra ${ }^{34}$. Outro estudo com 27 pacientes notou que o andexanet inverteu a atividade anticoagulante da apixabana e da rivaroxabana em poucos minutos após administração e durante a infusão da droga, sem evidência de efeitos tóxicos clínicos ${ }^{35}$. Segue em andamento um estudo de fase $3 b / 4$, ANNEXA- A\&R, os resultados das fases anteriores foram promissores ${ }^{36}$. Contudo, ainda carece de evidências suficientes sobre a real segurança e eficácia do andexanet alfa. E o seu uso ainda não está autorizado ${ }^{37}$.

\section{CONCLUSÃO}

Os NOACs abriram caminho para evolução e modernização no tratamento e prevenção de eventos cardioembólicos e tromboembólicos em paciente com FA. Ainda faltam evidências para embasar e esclarecer as indicações precisas para o uso de NOACs em grupos específicos de indivíduos. Além disso, médicos e pacientes precisam aprender a usar essas drogas de forma eficaz e segura, na prática clínica ${ }^{38}$. Dados de resultados de novos ensaios clínicos vêm sendo exponencialmente gerados para preencher as lacunas existentes. A esperança é de que, em um futuro próximo, questões ainda angustiantes, tais como necessidade de antídotos específicos para os inibidores diretos do fator Xa e melhor esclarecimento sobre qual o real risco de sangramento gastrointestinal dos AODs em relação à warfarina, sejam respondidas com mais precisão.

\section{TABELA}

Tabela 1. Comparações entre os Novos Anticoagulantes Orais

\begin{tabular}{|c|c|c|c|c|}
\hline & DABIGATRANA & RIVAROXABANA & APIXABANA & EDOXABANA \\
\hline Nome Comercial & Pradaxa $\AA$ & Xarelto $®$ & Eliquis ${ }^{\circledR}$ & Savysa ${ }^{\circledR}$ \\
\hline Indicações Aprovadas & $\begin{array}{l}\text { FA não valvar } \\
\text { Embolia sistêmica }\end{array}$ & $\begin{array}{l}\text { FA não valvar } \\
\text { Embolia sistêmica }\end{array}$ & $\begin{array}{l}\text { FA não valvar } \\
\text { Embolia sistêmica }\end{array}$ & $\begin{array}{l}\text { FA não valvar } \\
\text { Embolia sistêmica }\end{array}$ \\
\hline \multirow{2}{*}{$\begin{array}{l}\text { Mecanismo de Ação } \\
\text { Tempo de Pico }\end{array}$} & Inibidor direto da trombina & Inibidor do fator Xa & Inibidor do fator Xa & Inibidor do fator Xa \\
\hline & $\begin{array}{l}\text { 1h (atrasa para } 2 \mathrm{~h} \text { com } \\
\text { alimentação) }\end{array}$ & $2-4 h$ & 3- $4 \mathrm{~h}$ & $1-2 \mathrm{~h}$ \\
\hline \multirow[t]{3}{*}{ Biodisponibilidade } & \multirow[t]{3}{*}{$3 \%-7 \%$} & 10mg/dose: 80 a 100\% & \multirow[t]{3}{*}{ Aproximadamente $50 \%$} & \multirow[t]{3}{*}{$62 \%$} \\
\hline & & 20mg/dose: $66 \%$ & & \\
\hline & & Aumenta com alimentação & & \\
\hline $\begin{array}{l}\text { Ligação à Proteína } \\
\text { Plasmática }\end{array}$ & $35 \%$ & $92-95 \%$ & $\sim 87 \%$ & $55 \%$ \\
\hline \multirow[t]{4}{*}{$1 / 2$ vida no plasma } & $12-17 h$ & $5-9 h$ & \multirow[t]{4}{*}{$12 h(8-16 h)$} & \multirow[t]{4}{*}{$10-14 h$} \\
\hline & Idoso: $14-17 \mathrm{~h}$ & \multirow[t]{3}{*}{ Idoso: $11-13 h$} & & \\
\hline & IR leve a moderada: $15-18 \mathrm{~h}$ & & & \\
\hline & IR grave: $28 \mathrm{~h}$ & & & \\
\hline \multirow[t]{4}{*}{ Metabolismo } & $\begin{array}{l}\text { Hepático e hidrólise } \\
\text { plasmática para ativar a } \\
\text { dabigatrana }\end{array}$ & $\begin{array}{l}\text { Hepático: oxidação por } \\
\text { CYP3A4/5, CYP2J2; } \\
\text { hidrólise para inativar } \\
\text { metabólitos (51\%) }\end{array}$ & $\begin{array}{l}\text { Hepático: } 25 \% \\
\text { principalmente por } \\
\text { CYP3A4/5, menos por } \\
\text { CYP1A2, CYP2C8, CYP2C9, } \\
\text { CYP2C19, CYP2J2; } \\
\text { O-desmetilação e hidroxilação }\end{array}$ & $\begin{array}{l}\text { Mínima hidrólise de CYP3A4, } \\
\text { conjugação, oxidação }\end{array}$ \\
\hline & $\begin{array}{l}\text { Glicuronidação hepática para } \\
\text { metabólitos ativos }(<10 \%)\end{array}$ & Substrato P-gp & $\begin{array}{l}\text { Não há metabólitos } \\
\text { ativos circulando }\end{array}$ & $\begin{array}{l}\text { Metabólitos ativos (M-4, } \\
<10 \% \text { do pai) }\end{array}$ \\
\hline & \multirow[t]{2}{*}{ Substrato P-gp } & $\begin{array}{l}\text { Não há metabólitos ativos } \\
\text { importantes circulando }\end{array}$ & \multirow[t]{2}{*}{$\begin{array}{l}\text { Substrato de CYP3A4, P-gp, } \\
\text { BCRP }\end{array}$} & \multirow[t]{2}{*}{ P-gp substrato } \\
\hline & & Substrato de P-gp e ABCG2 (BCRP) & & \\
\hline
\end{tabular}




\begin{tabular}{|c|c|c|c|c|}
\hline \multirow[t]{2}{*}{ Excreção } & $\begin{array}{l}\text { Renal }(\sim 80 \%) \text { depois de } \\
\text { administração IV }\end{array}$ & $\begin{array}{l}\text { Renal (66\%):metabólitos 36\% } \\
\text { ativo, 30\% inativos. }\end{array}$ & Renal (27\%) & $\begin{array}{l}\text { Renal ( 50\%): } \\
\text { primariamente como droga } \\
\text { inalterada. }\end{array}$ \\
\hline & $\begin{array}{l}\text { Urina }(7 \%) \\
\text { Fezes }(86 \%)\end{array}$ & $\begin{array}{l}\text { Fezes (28\%): metabólitos } 7 \% \\
\text { ativos, } 21 \% \text { inativos. }\end{array}$ & $\begin{array}{l}\text { Excreção biliar e intestinal } \\
\text { direta }\end{array}$ & $\begin{array}{l}\text { Metabolismo e biliar/ } \\
\text { Liberação intestinal }\end{array}$ \\
\hline \multirow[t]{5}{*}{ Dose na FA não valvar } & $\begin{array}{l}\mathrm{ClCr}>30 \mathrm{~mL} / \mathrm{min}: 150 \mathrm{mg} \\
\text { 2x/dia }\end{array}$ & $\begin{array}{l}\mathrm{ClCr}>50 \mathrm{~mL} / \mathrm{min}: 20 \mathrm{mg} \\
\text { diariamente com refeição } \\
\text { noturna }\end{array}$ & $5 \mathrm{mg} 2 \mathrm{x} / \mathrm{dia}$ & $\begin{array}{l}\mathrm{ClCr}>50 \text { para } \leq 95 \mathrm{~mL} / \mathrm{min}: \\
60 \mathrm{mg} \text { diariamente }\end{array}$ \\
\hline & $\begin{array}{l}\mathrm{CICr} 15-30 \mathrm{~mL} / \mathrm{min}: 75 \mathrm{mg} \\
2 \mathrm{x} / \mathrm{dia}\end{array}$ & $\begin{array}{l}\mathrm{ClCr} 15-50 \mathrm{~mL} / \mathrm{min}: 15 \mathrm{mg} \\
\text { diariamente com refeição } \\
\text { noturna. }\end{array}$ & \multirow{4}{*}{$\begin{array}{l}2,5 \mathrm{mg} 2 \mathrm{x} / \mathrm{dia} \text {, se } 2 \text { das } 3 \\
\text { características: } \mathrm{Cr} \geq 1,5 \mathrm{mg} / \\
\text { dl; idade } \geq 80 \text { anos, peso } \\
\geq 60 \mathrm{Kg} \text {. }\end{array}$} & $\begin{array}{l}\mathrm{ClCr} 15-50 \mathrm{~mL} / \mathrm{min}: 30 \mathrm{mg} \\
\text { diariamente. }\end{array}$ \\
\hline & $\begin{array}{l}\mathrm{CICr}<15 \mathrm{~mL} / \mathrm{min} \text { ou na } \\
\text { diálise: não recomendado }\end{array}$ & \multirow[t]{3}{*}{$\begin{array}{l}\text { Não recomendado para } \mathrm{ClCr} \\
<15 \mathrm{~mL} / \mathrm{min} \text { ou na diálise }\end{array}$} & & \multirow[t]{3}{*}{$\begin{array}{l}\text { Não recomendado para } \mathrm{ClCr} \\
>95 \mathrm{~mL} / \mathrm{min} \text {. }\end{array}$} \\
\hline & $\begin{array}{l}\mathrm{CICr} 30-50 \mathrm{~mL} / \mathrm{min} \\
\text { concomitante a inibidores } \\
\text { P-gp: } 75 \mathrm{mg} 2 \mathrm{x} / \mathrm{dia}\end{array}$ & & & \\
\hline & $\begin{array}{l}\mathrm{CICr}<30 \mathrm{~mL} / \mathrm{min} \\
\text { concomitante com inibidores } \\
\text { P-gp: Evitar coadministração }\end{array}$ & & & \\
\hline \multirow[t]{2}{*}{$\begin{array}{l}\text { Comentários sobre dosagem } \\
\text { adicional }\end{array}$} & & $\begin{array}{l}\text { Evitar uso com pacientes com } \\
\text { moderada a grave disfunção } \\
\text { hepática (Child-Pugh classe } \\
\text { B/C) ou doença hepática com } \\
\text { coagulopatia }\end{array}$ & \multirow[t]{2}{*}{$\begin{array}{l}\text { Não recomendado em } \\
\text { pacientes com disfunção } \\
\text { hepática grave (Child-Pugh } \\
\text { classe C). }\end{array}$} & $\begin{array}{l}\text { Não recomendado com } \mathrm{ClCr} \\
<15 \mathrm{~mL} / \mathrm{min}\end{array}$ \\
\hline & & $\begin{array}{l}\text { Na dose de } 15-20 \mathrm{mg} \text {, junto } \\
\text { com alimento; na dose de } \\
10 \mathrm{mg}, \text { pode tomar com ou } \\
\text { sem alimento. }\end{array}$ & & $\begin{array}{l}\text { Não recomendado em } \\
\text { disfunção hepática moderada } \\
\text { a grave (Child-Pugh classe } \\
\text { B/C). }\end{array}$ \\
\hline \multirow[t]{4}{*}{ Medição Terapêutica } & Não requer de rotina & Não requer de rotina & Não requer de rotina & Não requer de rotina \\
\hline & $\begin{array}{l}\text { P/ detectar presença: TTPa, } \\
\text { ECT e TT }\end{array}$ & $\begin{array}{l}\text { P/ detectar presença: TP, } \\
\text { TTPa, atividade antifator } \mathrm{Xa.}\end{array}$ & $\begin{array}{l}\text { P/ detectar presença: TP, } \\
\text { TTPa, atividade antifator Xa }\end{array}$ & $\begin{array}{l}\text { TP prolongados, TTPa, } \\
\text { atividade antifator Xa }\end{array}$ \\
\hline & $\begin{array}{l}\text { TTPa }>2,5 \text { o tempo controle } \\
\text { pode indicar anticoagulação }\end{array}$ & \multirow[t]{2}{*}{$\begin{array}{l}\text { Função renal, hemograma } \\
\text { anual, função hepática. }\end{array}$} & \multirow[t]{2}{*}{$\begin{array}{l}\text { Função renal, hemograma } \\
\text { anual. }\end{array}$} & \multirow[t]{2}{*}{$\begin{array}{l}\text { Função renal, hemograma } \\
\text { anual. }\end{array}$} \\
\hline & $\begin{array}{l}\text { Função renal, hemograma } \\
\text { anual. }\end{array}$ & & & \\
\hline
\end{tabular}

FA (Fibrilação Atrial); IR (Insuficiência Renal); P-gp (Glicoproteína-P); ECT(Tempo de Coagulação de Ecarina); TT (Tempo de Trombina); TP (Tempo de Protrombina).

*Tabela adaptada - RAVAL, A. N. et al. Management of Patients on Non-Vitamin K Antagonist Oral Anticoagulants in the Acute Care and Periprocedural Setting: A Scientifc Statement From the American Heart Association. Circulation, 135:00-00, fev., 2017.

\section{REFERÊNCIAS}

1. Outes, A. G. et al. Causes of Death in Anticoagulated Patients With Atrial Fibrillation.Journal of the American College of Cardiology. Madrid, v. 6 8, n. 23 , dez., 2016.

2. Bpharm, A. S. et al. Cost Effectiveness of Oral Anticoagulants for Ischemic Stroke Prophylaxis Among Nonvalvular Atrial Fibrillation Patients. Stroke,dez., 2016.

3. Verheugt, F. W. A.; Granger, C. B. Novel antithrombotic agents 3 . Oral anticoagulants for stroke prevention in atrial fibrillation: current status, special situations, and unmet needs. The Lancet.Amsterdam, mar., 2015.
4. Molina, J. E.; Naeimi, P. S.; Razavi, M. What's New in Anticoagulation. Texas Heart Institute Journal. Houston, v. 43, n. 5, out.,2016.

5. Garcia, D. A; Crowther, M. Management of bleeding in patients receiving direct oral anticoagulants. UpToDate. Disponível em: <https://www.uptodate. com>. Acesso em 26 de julho de 2017.

6. Blair, H. A., Keaing, G. M. Dabigatran Etexilate: A Review in Nonvalvular Atrial Fibrillation. Adis drug evalution, Nova Zelândia, fev., 2017.

7. Connoly, S. J. et al. Dabigatran versus Warfarin in Patients with Atrial Fibrillation.N. Engl. J. Med. Massachusetts, v. 361, n. 12, p. 1139-51, set., 2009.

8. Plitt, A.; Mcguire, D. K.; Giugliano, R. P. Comment 
\& Respose.JAMA Cardiology, jun., 2017. Disponível em: <http://cardiology.jamanetwork.com/ by>. Acesso em 26 de julho de 2017.

9. Graham, D. J. et al. Cardiovascular, Bleeding, and Mortality Risks in Elderly Medicare Patients Treated With Dabigatran or Warfarin for Nonvalvular Atrial Fibrillation. Circulation, jan., 2015.

10. Sharma, M. et al.Efficacy and Harms of Direct Oral Anticoagulants in the Elderly for Stroke Prevention in Atrial Fibrillation and Secondary Prevention of Venous Thromboembolism: Systematic Review and Meta-Analysis. Circulation, Londres, maio, 2015.

11. Majeed, A. et al. Management and Outcomes of Major Bleeding DuringTreatment With Dabigatran or Warfarin. Circulation, Canada, p. 2325-37 nov., 2013.

12. Chan, Y. H. et al.Cardiovascular, Bleeding, and Mortality Risks of Dabigatran in Asians With Nonvalvular Atrial Fibrillation. Stroke, Taiwan p. 441-53, fev., 2016.

13. Lau, W. C. Y. et al.Association Between Dabigatran vs Warfarin and Risk of Osteoporotic Fractures Among Patients With Nonvalvular Atrial Fibrillation. JAMA, v. 317, n. 11, p.1151-58, mar., 2017.

14. Chales, V. P. et al. Idarucizumab for Dabigatran Reversal.N. Engl. J. Med. Massachusetts, v. 373, p. 511-20, ago., 2015.

15. Francine J. Azevedo, F. J.; Uchôa, A. F. T.; Costa, T. D. et al. Papel da Glicoproteína-P na Farmacocinética e nas Interações Medicamentosas. Revista Brasileira de Farmacologia, v. 90, n. 4, p. 321-326, ago., 2009.

16. Raval, A. N. et al. Management of Patients on Non-Vitamin K Antagonist Oral Anticoagulants in the Acute Care and Periprocedural Setting:A Scientifc Statement From the American Heart Association. Circulation, 135:00-00, fev., 2017.

17. Patel, M. R. et al. Rivaroxaban versus Warfarin in Nonvalvular Atrial Fibrillation.N. Engl. J. Med. Massachusetts, v. 365, n. 10, p. 883-91, set., 2011.

18. Granger, C. B. et al. Apixaban versus Warfarin in Patients with Atrial Fibrillation.N. Engl. J. Med. Massachusetts, v. 365, n. 11, p. 981-92, set., 2011.

19. Giugliano, R. P. et al.Edoxaban versus Warfarin in Patients with Atrial Fibrillation.N. Engl. J. Med. Massachusetts, v. 369, n. 22, p. 2093-104, nov., 2013.

20. Ruff, C. T. et al. Association between edoxaban dose, concentration, anti-Factor Xa activity, and outcomes: an analysis of data from the randomised, double-blind ENGAGE AF-TIMI 48 trial. The Lancet, Boston, mar., 2015.

21. Ruff, C. T. et al. Comparison of the efficacy and safety of new oral anticoagulants with warfarin in patients with atrial fi brillation: a meta-analysis of randomised trials. The Lancet, dez., 2013.

22. Janzic, A., Kos, M. Cost Effectiveness of Novel Oral Anticoagulants for Stroke Prevention in Atrial Fibrillation Depending on the Quality of Warfarin Anticoagulation Control.Pharmaco Economics, Ljubljana, dez., 2014.

23. Diener, H. C. et al. Choosing a particular oral anticoagulante and dose for stroke prevention in individual patients with non-valvular atrial fibrillation: part 1 . European Heart Journal, fev., 2016....

24. Cannon, C. P. et al. Design and Rationale of the RE-DUAL PCI Trial: A Prospective, Randomized, Phase $3 b$ Study Comparing the Safety and Efficacy of Dual Antithrombotic Therapy With Dabigatran Etexilate Versus Warfarin Triple Therapy in Patients With Nonvalvular Atrial Fibrillation Who Have Undergone Percutaneous Coronary Intervention With Stenting. Clinical Cardiology, jun., 2016.

25. Gibson, C. M. et al. Prevention of Bleeding in Patients with Atrial Fibrillation Undergoing PCI.N. Engl. J. Med. Massachusetts, v. 375, n. 25, dez., 2016.

26. Povsic, T. J. et al. A randomized trial to compare the safety of rivaroxaban vs aspirin in addition to either clopidogrel or ticagrelor in acute coronary syndrome: The design of the GEMINI-ACS-1 phase II study.American Heart Journal, v. 174, P. 120-128, jan., 2016.

27. Santagelli, P. et al. Ablation of atrial fibrillation under therapeutic warfarin reduces periprocedural complications: evidence from a meta-analysis.Circ Arrhythm Electrophysiol, v. 5, p. 302-311, abr., 2012.

28. Camm, A. J. et al. ESC Committee for Practice Guidelines (CPG). 2012 focused update of the ESC Guidelines for the management of atrial fibrillation: an update of the 2010 ESC Guidelines for the management of atrial fibrillation - developed with the special contribution of the European Heart Rhythm Association. European Heart Journal, v. 33, p.2719-47, abr., 2014.

29. Hohnloser, S. H; Cann, A. J. Safety and efficacy of dabigatran etexilate during cateter ablation of atrial fibrillation: a meta-analysis of the literature.Europace, v. 15, p. 1407-1411, fev., 2014.

30. Calkins, $H$. et al.Uninterrupted Dabigatran versus Warfarin for Ablation in Atrial Fibrillation.N. Engl. J. Med. Massachusetts, mar., 2017.

31. Nagao, T. et al. Efficacy and safety of apixaban in the patients undergoing the ablation of atrial fibrillation. Pacing Clin Electrophysiol, v. 38, p. 155-163, fev., 2015. 
32. Diener, H. C. et al. Choosing a particular oral anticoagulante and dose for stroke prevention in individual patients with non-valvular atrial fibrillation: part 2. European Heart Journal, fev., 2016.

33. Siegal, D. M. et al. How I treat target-specific oral anticoagulant-associated bleeding.Blood, v. 123, n.8, p.1152-58, fev., 2014.

34. Connolly, S. J. et al. Andexanet Alfa for Acute Major Bleeding Associated with Factor Xa Inhibitors.N. Engl. J. Med. Massachusetts, v. 375, p. 1131-41, ago., 2016.

35. Siegal, D. M. Andexanet Alfa for the Reversal of Factor Xa Inhibitor Activity.N. Engl. J. Med. Massachusetts, v.373, n. 25, p. 2413-24, dez., 2015..

36. Florie, M. et al. Andexanet Alfa: An Investigational Universal Antidote for Reversal of Anticoagulation of Factor Xa Inhibitors in Healthy Human Volunteers. Congress of Neurological Surgeons, v. 63, n. 1, ago., 2016.

37. Magalhães, L. P. et al. II Diretrizes Brasileiras de Fibrilação Atrial. Revista da Sociedade Brasileiras de Cardiologia. Rio de Janeiro, v. 106, n. 4, supl. 2, abr., 2016.

38. Heidbuchel, H. et al. Updated European Heart Rhythm Association Practical Guide on the use of non-vitamin $\mathrm{K}$ antagonista anticoagulants in patients with non-valvular atrial fibrillation. European Society of Cardiology, ago., 2015.

1- Serviço de Neurologia do HSI

Endereço para correspondência:

itananaiara@yahoo.com.br 